\title{
Characterization of Nopal Nanoparticles by Electron Microscopy Techniques
}

Jocelyn Madrigal-Acevedo ${ }^{1}$, Jorge Chanona-Pérez ${ }^{1}$, Leticia Manuel-Apolinar ${ }^{2}$, Héctor Calderón ${ }^{3}$ and Issis Claudette Romero Ibarra ${ }^{4}$

1. Departamento de Ingeniería Bioquímica, Escuela Nacional de Ciencias Biológicas, Instituto Politécnico Nacional, Mexico City, Mexico.

2. Unidad de Investigación Médica en Enfermedades Endócrinas, Hospital de Especialidades, Centro Médico Nacional Siglo XXI, Instituto Mexicano del Seguro Social, Mexico City, Mexico.

3. Escuela Superior de Física y Matemáticas, Instituto Politécnico Nacional, Mexico City, Mexico.

4. Unidad Profesional Interdisciplinaria en Ingeniería y Tecnologías Avanzadas, Instituto Politécnico Nacional Mexico City, Mexico.

Nopal (Opuntia ficus-indica) is rich in dietary fiber, vitamin C, micronutrients and polyphenols; so that, is widely used in traditional Mexican medicine as a treatment to reduce the inflammation in pathologies related to dysmetabolic syndrome (Maki-Díaz et al., 2015; Muñoz et al., 2014; Armijos et al., 2013). The tendency in food science is improve the physicochemical and nutraceutical properties for which nanotechnology is applied. High impact milling is an attractive method to obtain biological nanoparticles, without using chemical reagents and with minimal damage to the bioactive substances contained in the material. Nanoparticulation increases surface area, availability and reactivity of its bioactive components, which allow a large diffusion rate and assimilation to target organs for improve their functionality and nutraceutical activity. For the characterization of biological nanoparticles electron microscopy is helpful, but the use of low voltages and dosses are required. The aim of this work was obtained nopal nanoparticles (NNP) by high impact milling and characterize by electron microscopy techniques. Nopal powder was obtained from local producer (INMA, Mexico City). This powder was used to obtain NPP by a ball mill, planetary type (Premium line pulverisette 7, Fritsch, Germany). Particle sizes were obtained by particle analyzer (Zetasizer nano s90). Overall morphology of the nopal powder INMA and NNP were observed with a scanning electron microscope (SEM, Quanta 3D FEG; FEI, Hillsboro, OR, USA) at 10kV and 0.8-1.0 mbar and a transmission electron microscope (TEM, JEM-2100, Jeol, USA). Surface area was determined by BET method and biological studies in rats were performed to evaluate the nutraceutical effect of nanoparticles. The particle sizes determined in both powders were $1.8 \pm 0.6 \mu \mathrm{m}$ (INMA) and $92 \pm 26.39 \mathrm{~nm}(\mathrm{NNP})$. Figure 1 shows the SEM images of INMA and NNP powders, it is observed that INMA and NNP have an irregular shape. NNP showed a tendency to agglomerate, which is common in nanomaterials produced by milling. EDS spectra indicate the presence of trace elements as $\mathrm{Al}, \mathrm{Ca}$ and $\mathrm{K}, \mathrm{Ca}$ is associated with the oxalate crystals usually contain cacateceae species, the presence of $\mathrm{Mg}$ in NNP is associated with the prosthetic group of the chlorophylls, TEM images (Figure 2) provide a better visualized of INMA and NNP powders and evidenced the presence of crystalline structures (see diffraction patterns) in INMA powder maybe correspond to oxalate crystals, while NNP had amorphous structure caused by milling process. Specific surface area of INMA powder was $0.737 \mathrm{~m}^{2} / \mathrm{g}$, while milling process yielded NNP with $3.264 \mathrm{~m}^{2} / \mathrm{g}$, this represent an increase of 4.5 times of surface area in nanoparticulated powders. In biological experiments, the obese rats NNP had a similar hypoglycemic effect to INMA powder but with a dose of NNP five times lower. The irregular shape and amorphous stage of NNP, the exposition of $\mathrm{Mg}$ evidenced by electron microscopy, and the increase its specific surface area suggest that the milling process exposes more bioactive compounds, that provide an explanation of increase of hypoglycemic effect of the NNP. Electron microscopy was useful to characterize biological powders. 
References:

[1] G Maki-Díaz et al., Agrociencia 49 (2015), p. 31.

[2] E Neri-Torres et al., Microsc Microanal. 22(4) (2016), p. 887.
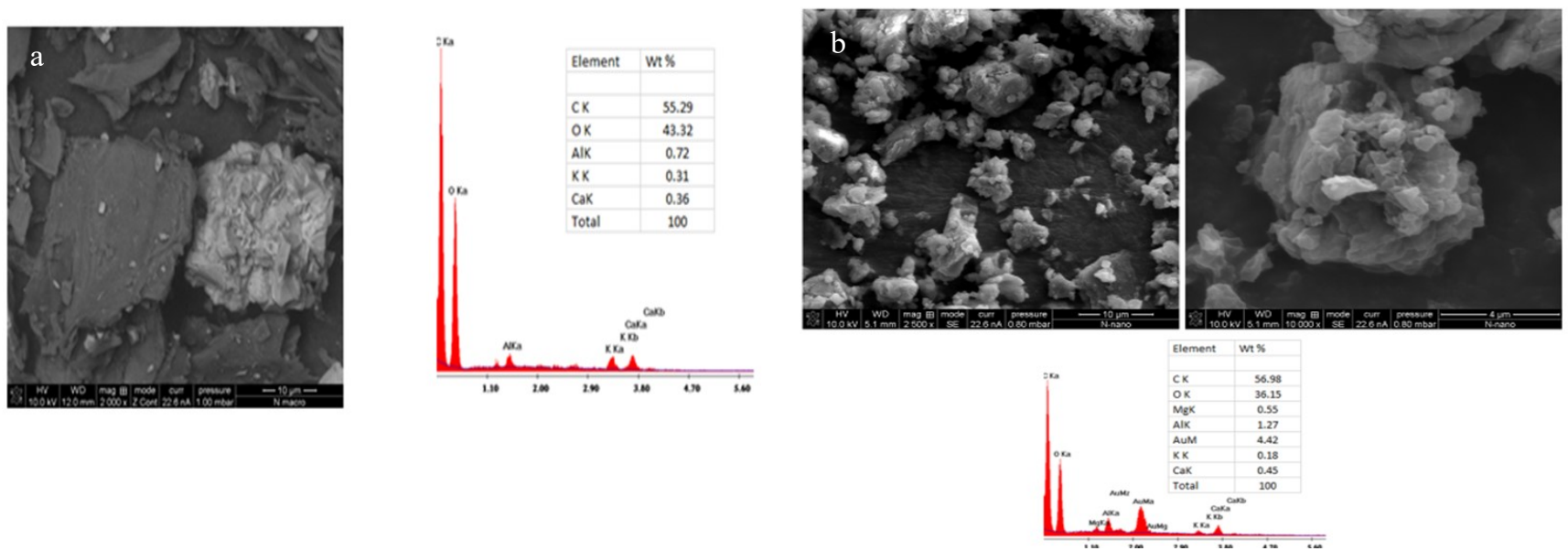

Figure 1. SEM images show the particles of INMA (a) and NNP (b) powders and their EDS spectra.
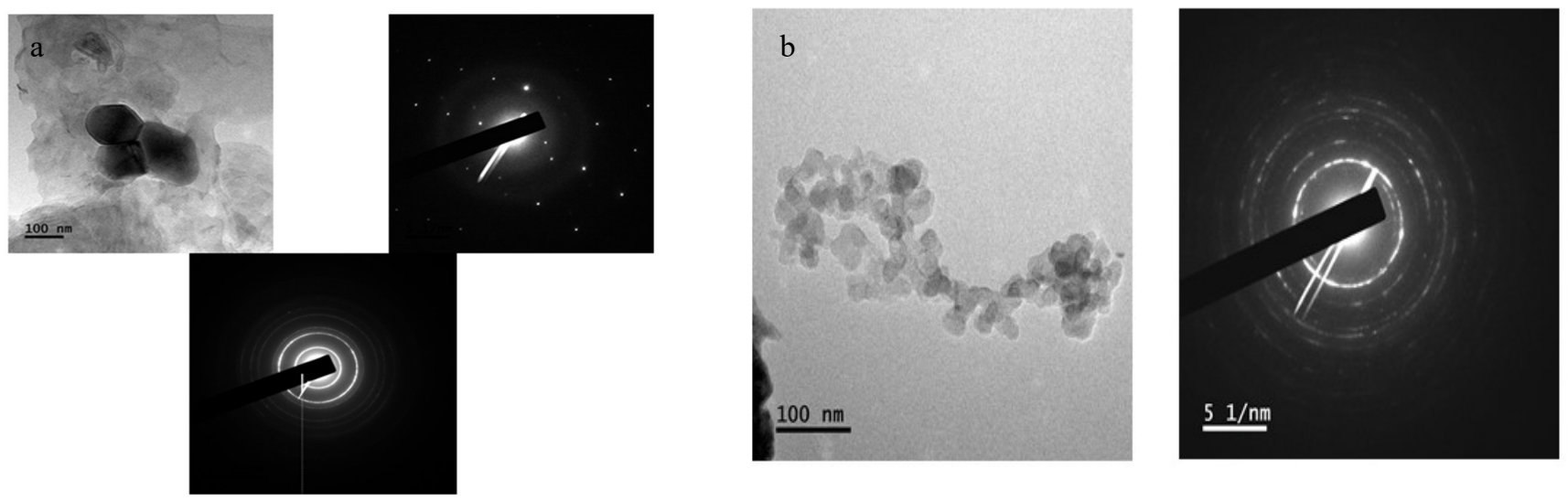

Figure 2. TEM images of INMA (a) and NNP (b) powders and with diffraction patterns in amorphous and crystalline zones. 